\title{
Grupo Genético e Heterose na Produção de Leite de Vacas de Corte Submetidas a Diferentes Sistemas de Alimentação
}

\author{
João Restle ${ }^{1}$, Dilceu Borges de Senna ${ }^{2}$, Paulo Santana Pacheco ${ }^{3}$, João Teodoro Padua ${ }^{4}$, \\ Ricardo Zambarda Vaz ${ }^{5}$, Patrícia Alessandra Meneguzzi Metz ${ }^{6}$
}

RESUMO - O experimento foi conduzido para se avaliar a produção de leite de vacas de dois sistemas de acasalamento, puras Charolês $(\mathrm{CH})$ e Nelore (NE) e mestiças F $1 \frac{1}{2} \mathrm{CH}$ 1/2 $\mathrm{NE}$ e $1 / 2 \mathrm{NE} 1 / 2 \mathrm{CH}$, submetidas a diferentes sistemas de alimentação, e medir a heterose resultante. A produção de leite foi determinada aos 14, 42, 70 e 90 dias, sendo esta última coincidente com o desmame. Foram utilizadas 86 vacas, submetidas a um dos seguintes sistemas de alimentação: PN: vacas mantidas exclusivamente em pastagem nativa; PC + PN: vacas mantidas em pastagem cultivada (PC) de 15 de julho a 15 de setembro e o restante do tempo em PN; e PN + PC: vacas mantidas em PC de 15 de setembro a 15 de novembro e o restante do tempo em PN. A PC foi composta por aveia (Avena sativa), azevém (Lollium multiflorum) e trevo vesiculoso (Trifolium vesiculosum). Não houve diferença significativa entre os sistemas de alimentação PC + PN e PN + PC, agrupando-se os dois em um único sistema (PNC). Vacas mantidas em PN apresentaram menor produção média diária (3,93 vs. 5,46 L) e total de leite (291,4 vs. 401,7 L) e maior redução da produção (39 vs. 27\%) que as mantidas em PNC. Não houve efeito do grupo genético da vaca dentro do sistema de acasalamento sobre as produções média e total de leite. Vacas mestiças F1 apresentaram maior produção de leite durante toda a lactação, de modo que a heterose para produção média diária de leite foi mais expressiva nas vacas mantidas em condições mais baixas de alimentação, exclusivamente em $\mathrm{PN}(92,4 \%)$, que nas vacas que tiveram acesso à pastagem cultivada PNC $(18,1 \%)$. A persistência da lactação foi maior nas vacas filhas de touros NE que nas filhas de touros $\mathrm{CH}$, assim como nas vacas puras em relação às mestiças $\mathrm{F} 1$.

Palavras-chave: Charolês, cruzamento, desmame precoce, Nelore, pastagem cultivada, pastagem nativa

\section{Genetic Group and Heterosis on Milk Yield of Beef Cows Submitted to Different Feeding Systems}

\begin{abstract}
The objective of this trial was to evaluate the milk yield of cows of two breeding systems, straightbreds Charolais $(\mathrm{CH})$ and Nellore (NE) and, crossbreds $\mathrm{F} 1 \frac{1}{2} \mathrm{CH} 1 / 2 \mathrm{NE}$ and $1 / 2 \mathrm{NE} 1 / 2 \mathrm{CH}$, submitted to different feeding systems, as well as measure the resultant heterosis. The milk yield was determined at 14, 42, 70 and 90 days, being this last one coincident with weaning. Eightysix cows were used, and submitted to one of the following feeding systems: NP: cows maintained exclusively on native pasture (NP); $\mathrm{CP}+\mathrm{NP}$ : cows maintained on cultivated pasture (CP) from July $15^{\text {th }}$ until September $15^{\text {th }}$ and the rest of the time on NP; and NP + CP: cows maintained on CP from September $15^{\text {th }}$ until November $15^{\text {th }}$, and the rest of the time on NP. CP was composed by oat (Avena sativa), ryegrass (Lollium multiflorum) and clover (Trifolium vesiculosum). Since no significant difference was verified between $\mathrm{CP}+\mathrm{NP}$ and NP $+\mathrm{CP}$ feeding systems, they were combined in one feeding system (NCP). Cows maintained on NP showed lower average daily (3.93 vs. $5.46 \mathrm{~L})$ and total milk yield (291.4 vs. $401.7 \mathrm{~L})$, in relation to cows maintained on NCP, as well as displayed higher reduction of the milk yield ( $39 \mathrm{vs.} 27 \%$, respectively). Average daily and total milk yield were not affected by cow genetic group within breeding system. F1 cows showed higher milk yield during the whole lactation period, being the heterosis for average daily milk yield more expressive for cows maintained on lower feeding conditions, exclusively on NP (92.4\%) in relation to cows with access to NCP (18.1\%). The lactation persistency was higher for cows sired by NE bulls than for cows sired by CH bulls, and for straightbreds in relation to $\mathrm{F} 1$ cows.
\end{abstract}

Key Words: Charolais, crossbreeding, early weaning, Nellore, cultivated pasture, native pasture

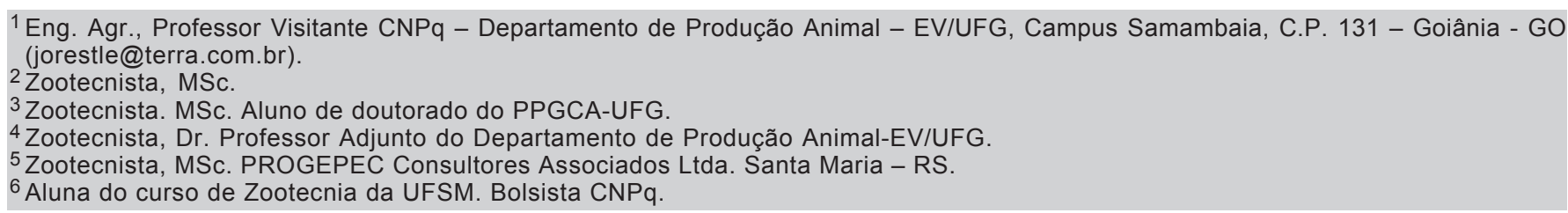




\section{Introdução}

A produção de leite em vacas de corte tem grande importância no sistema de produção de bovinos de corte graças à correlação positiva existente entre esta e o peso ao desmame do bezerro, conforme demonstrado nos estudos de Melton et al. (1967), Rutledge et al. (1971), Ribeiro \& Restle (1991), Albuquerque et al. (1993) e Restle et al. (2004). Neste sentido, propriedades que buscam a intensificação do sistema de produção devem priorizar vacas de corte com maior produção de leite, o que refletirá positivamente na produção de bezerros com maiores pesos ao desmame.

Contudo, deve-se entender os principais fatores que afetam a produção de leite de vacas de corte e, conseqüentemente, interferem no desempenho dos bezerros. Entre os fatores ambientais, destaca-se o nível nutricional ao qual os animais estão submetidos. Entre os genéticos, o mais importante é o grupo genético, além do tamanho corporal da vaca.

Quanto ao efeito nutricional, estudos conduzidos comprovam o seu reflexo positivo na produção de leite das vacas (Dunn et al., 1965; Bond \& Wiltbank, 1970; Polli \& Lobato, 1985). É possível obter acréscimos na produção planejando a estação de parição dos animais, que deve coincidir com os períodos de maiores disponibilidades de matéria seca (Boggs et al., 1980; Alencar et al., 1988); reduzindo a lotação animal/ha (Quadros \& Lobato, 1997); ou utilizando diferentes seqüências de pastagem (Ribeiro \& Restle, 1991; Restle et al., 2003). Contudo, em função dos custos elevados das pastagens cultivadas de inverno, há necessidade do estudo de períodos curtos de pastejo, no pré- e/ou pós-parto, objetivando potencializar seu uso, com a possibilidade do pastejo de outras categorias de bovinos, e verificar seus reflexos na produção de leite das vacas em comparação com a pastagem nativa.

Quanto ao efeito genético, trabalhos que compararam diferentes genótipos indicaram maiores produções para vacas de maior porte, como os de Alencar et al. (1993) e Cruz et al. (1997), que avaliaram as raças Canchim e Nelore, e Ribeiro et al. (1991), que trabalharam com vacas Charolês e Aberdeen Angus, e Melton et al. (1967), com animais Charolês, Aberdeen Angus e Hereford.

Estudos apontam para o benefício do cruzamento no desempenho de animais terminados em confinamento (Koger, 1973; Restle et al., 1995; Restle et al., 2001) e na redução da idade à puberdade
(Restle et al., 1999a). Visando a produção de leite, os resultados também foram positivos (Reynolds et al., 1978; Cundiff et al., 1974; Kress et al., 1990; Cerdótes et al., 2004). No entanto, poucos são os trabalhos que avaliaram a heterose para produção de leite ao longo do período de lactação, em diferentes sistemas de alimentação.

Neste estudo, objetivou-se avaliar a produção de leite de vacas de corte de dois sistemas de acasalamento puras Charolês $(\mathrm{CH})$ ou Nelore (NE) e mestiças F1 $1 / 2 \mathrm{CH} 1 / 2 \mathrm{NE}$ ou $1 / 2 \mathrm{NE} 1 / 2 \mathrm{CH}$, submetidas a diferentes sistemas de alimentação representados por pastagem nativa, exclusivamente, ou sua associação temporária com pastagem cultivada, e medir a heterose resultante.

\section{Material e Métodos}

O experimento foi conduzido no Setor de Bovinocultura de Corte do Departamento de Zootecnia da Universidade Federal de Santa Maria, em Santa Maria - RS, localizado na Depressão Central, a 153 m de altitude, que, segundo classificação de Köppen, apresenta clima subtropical úmido (cfa) (Moreno, 1961). A temperatura média anual varia de 14,3 a $25,2^{\circ} \mathrm{C}$, com média das mínimas de $9,7^{\circ} \mathrm{C}$ no mês de agosto e média das máximas de $29,9^{\circ} \mathrm{C}$ no mês de janeiro e possíveis geadas de abril a outubro. A média anual da umidade relativa do ar é de $73 \%$ e a precipitação, de 1650,9 mm (Rio Grande do Sul, 1979).

Foram utilizadas 86 vacas, selecionadas ao acaso no mesmo rebanho experimental, com idade de três a dez anos, dos grupos genéticos Charolês $(\mathrm{CH})$, Nelore (NE), $1 / 2 \mathrm{CH} 1 / 2 \mathrm{NE}$ e $1 / 2 \mathrm{NE} 1 / 2 \mathrm{CH}$, que produziram bezerros puros $\mathrm{CH}$ ou $\mathrm{NE}$, ou mestiços $1 / 2 \mathrm{CH}$ $1 / 2 \mathrm{NE}, 3 / 4 \mathrm{CH} 1 / 4 \mathrm{NE}$ (filhos de touros $\mathrm{CH}$ ) ou $1 / 2 \mathrm{NE}$ $1 / 2 \mathrm{CH}, 3 / 4 \mathrm{NE} 1 / 4 \mathrm{CH}$ (filhos de touros $\mathrm{NE}$ ), desmamados aos 90 dias pós-parto.

Os animais foram submetidos aos seguintes sistemas de alimentação: PN: vacas mantidas exclusivamente em pastagem nativa; $\mathrm{PC}+\mathrm{PN}$ : vacas mantidas em pastagem cultivada, composta por aveia (Avena sativa), azevém (Lollium multiflorum) e trevo vesiculoso (Trifolium vesiculosum) durante o período de 15 de julho a 15 de setembro e o restante do período experimental em pastagem nativa; e PN + PC: vacas mantidas em pastagem cultivada composta por aveia, azevém e trevo vesiculoso durante o período de 15 de setembro a 15 de novembro, e o restamte do período em pastagem nativa. 
No momento da parição, os animais foram sorteados nos tratamentos de acordo com o grupo genético e a idade da vaca.

O período de parição foi de 15 de setembro a 15 de dezembro. Os bezerros foram obtidos de inseminação artificial (período de 45 dias) e de monta natural (período de 45 dias), utilizando-se, para inseminação, o sêmen de oito touros $\mathrm{CH}$ e NE e, para o repasse, quatro touros $\mathrm{CH}$ e quatro NE. Os touros que produziram os bezerros $\mathrm{CH}$ foram os mesmos que produziram os $1 / 2 \mathrm{CH} \quad 1 / 2 \mathrm{NE}$ e $3 / 4 \mathrm{CH} \quad 1 / 4 \mathrm{NE}$ e os que produziram os bezerros NE, os mesmos que produziram os $1 / 2 \mathrm{NE} 1 / 2 \mathrm{CH}$ e $3 / 4 \mathrm{NE} 1 / 4 \mathrm{CH}$.

A lotação foi de 1,92 UA/ha na pastagem cultivada e de $0,8 \mathrm{UA} / \mathrm{ha}$ na pastagem nativa. A massa de forragem disponível foi avaliada por meio da técnica da dupla amostragem (Wilm et al., 1944). Mensalmente, foram coletadas amostras da forragem, cortada rente ao solo, em uma área de $0,25 \mathrm{~m}^{2}$, para posterior determinação da matéria seca (MS), em estufa de ventilação forçada, a $55^{\circ} \mathrm{C}$, por $72 \mathrm{~h}$, da digestibilidade in vitro da matéria orgânica (DIVMO), conforme Tilley \& Terry (1963), e da proteína bruta (PB), pelo método micro kjeldahl, segundo AOAC (1984).

O método utilizado para determinar a produção de leite foi o direto, com ordenha manual. As avaliações foram realizadas aos 14, 42, 70 e 90 dias pós-parto e apresentaram desvio de mais ou menos três dias de acordo com a data de parição. A metodologia empregada foi a descrita por Ribeiro et al. (1991) e Restle et al. (2003). As vacas eram separadas de seus bezerros pela manhã (um dia antes da ordenha) e soltas em piquete anexo. Por volta das $18 \mathrm{~h}$, eram recolhidas novamente até o centro de manejo, permanecendo com o bezerro por 30 minutos, tempo suficiente para esvaziar o úbere. Após mamarem, os bezerros eram separados novamente das vacas e ficavam contidos no centro de manejo, ao passo que as vacas eram soltas em piquete com pasto e água. No dia seguinte, às $6 \mathrm{~h}$, iniciava-se a ordenha. Para realização da ordenha, a vaca era contida pelo pescoço e a pata traseira esquerda era amarrada com corda, recebendo então, 3 U.I. de ocitocina via intramuscular, a fim de se facilitar a descida do leite. A ordenha foi realizada, após o úbere ser lavado e massageado, apenas nos dois quartos do lado direito, durante, aproximadamente, 5 minutos. A produção obtida em litros (L) era multiplicada por dois, para se obter a produção do úbere, que também era corrigida para 24 horas.
O delineamento experimental adotado foi o inteiramente casualisado e os dados foram submetidos à análise de variância e aos testes $\mathrm{F}$ e Tukey quando o $\mathrm{F}$ foi significativo a $5 \%$, por meio do programa estatístico SAS (1997). A partir da análise estatística preliminar, verificou-se que não houve diferença significativa entre os sistemas de alimentação PC + PN e PN + PC (Tabela 1), decidindo-se por agrupálos em um único sistema (PNC). Foram analisadas a produção de leite aos 14, 42, 70 e 90 dias e as produções média e total, utilizando-se o seguinte modelo matemático:

$$
\begin{gathered}
\Psi_{i j k l m n}=\mu+\mathrm{NT}_{i}+\mathrm{IV}_{j}+\mathrm{PAST}_{k}+\mathrm{SAV}_{l}+ \\
\mathrm{GGV}_{m}\left(\mathrm{SAV}_{l}+\left(\mathrm{PAST}^{*} \mathrm{GGV}[\mathrm{SAV}]\right)_{k m l}+\right. \\
\left(\mathrm{PAST}^{*} \mathrm{SAV}\right)_{k l}+\varepsilon_{i j k l m n},
\end{gathered}
$$

em que: $\Psi_{i j k l m n}=$ variáveis dependentes; $\mu=$ média geral de todas as observações; $\mathrm{NT}_{i}=$ efeito da covariável "ordem de nascimento do bezerro" de ordem "i"; $\mathrm{IV}_{j}=$ efeito da covariável idade da vaca de ordem "j"; $\mathrm{PAST}_{k}=$ efeito do sistema de alimentação de ordem " $k$ ", em que 1 = pastagem nativa $\mathrm{PN} ; 2$ = pastagem nativa mais cultivada (PNC); $\mathrm{SAV}_{l}=$ efeito do sistema de acasalamento que originou as vacas de ordem "l", sendo $1=$ puras e 2 = mestiças F1; $\mathrm{GGV}_{m}(\mathrm{SAV})_{l}=$ efeito do grupo genético da vaca de ordem " $\mathrm{m}$ " sendo $1=$ Charolês $(\mathrm{CH}) ; 2=$ Nelore $(\mathrm{NE}) ; 3=1 / 2 \mathrm{CH} \quad 1 / 2 \mathrm{NE}$ e $4=1 / 2 \mathrm{Ne}$ $1 / 2 \mathrm{CH}$ dentro do sistema de acasalamento de ordem "l"; (PAST*GGV[SAV]) $)_{k m l}=$ interação entre k-ésimo sistema de alimentação e m-ésimo grupo genético da vaca dentro do l-ésimo sistema de acasalamento; $\left(\mathrm{PAST}^{*} \mathrm{SAV}\right)_{k l}=$ interação entre $\mathrm{k}$-ésimo sistema de alimentação e 1-ésimo sistema de acasalamento; $\varepsilon_{i j k l m n}=$ erro aleatório residual, $\operatorname{NID}\left(0, \sigma^{2}\right)$.

O grupo genético do bezerro não foi incluído no modelo, pois está parcialmente confundido com o grupo genético da vaca.

Para a variável persistência da lactação, os dados não apresentaram distribuição normal (Teste de Shapiro-Wilk) e foram submetidos à transformação radicial (raiz quadrada da variável).

Foi calculada a heterose $(\mathrm{H})$ para as características avaliadas por meio da seguinte fórmula: $\mathrm{H}(\%)=([$ média dos animais cruzados/média dos animais puros] -1$) * 100$. 


\section{Resultados e Discussão}

$\mathrm{Na}$ Tabela 1, encontram-se os valores médios referentes às produções diária e total de leite das vacas, em litros, de acordo com o sistema de alimentação e os dias de lactação.

Analisando a produção de leite das vacas aos 42 e 70 dias, verifica-se que houve similaridade entre os dois sistemas de alimentação avaliados. As diferenças entre os sistemas, na ordem de 23\% (42 dias) e $22 \%$ (70 dias), aumentaram para 44\% (14 dias) e 57\% (90 dias). No entanto, as vacas mantidas em pastagem nativa apresentaram menor produção diária de leite que as mantidas em pastagem nativa mais pastagem cultivada. A diferença média verificada foi significativa e na ordem de $39 \%$ (3,93 vs. 5,46 L/dia, respectivamente), similar à verificada para a produção total de leite, de 401,7 vs. 291,4 litros ( $\mathrm{P}=0,0043)$. A maior produção das vacas que, além da pastagem nativa tiveram acesso a pastagem cultivada (PNC) é explicada pela melhor qualidade de $\mathrm{PNC}$ em relação à pastagem nativa, uma vez que a disponibilidade de matéria seca (MS) foi menor (1108 vs. $1636 \mathrm{~kg} \mathrm{MS} / \mathrm{ha}$ ). Os valores da digestibilidade in vitro da matéria orgânica (DIVMO) e do teor de proteína bruta (PB) foram de 63,2 e 11,7\%, para pastagem cultivada, e de 40,2 e 5,9\%, para pastagem nativa.

Este resultado demonstra a vantagem do uso de períodos curtos de pastagem cultivada na produção de leite em vacas de corte. Neste estudo, foi aproveitada apenas uma parte do potencial produtivo da pastagem cultivada, uma vez que seu ciclo poderia ultrapassar 150 dias de pastejo. Se for considerado um sistema de produção de ciclo completo (cria, recria e terminação), esta pastagem ainda poderia ser utilizada por outras categorias, potencializando seu uso e reduzindo o custo de sua utilização.
Em condições sul-brasileiras, Restle et al. (2003) verificaram que, até os 98 dias de lactação, de um total de 182 dias, vacas mantidas em pastagem cultivada produziram $10,1 \%$ mais leite que as mantidas em pastagem nativa (5,26 vs. 4,77 L/dia). Avaliando o uso de suplemento energético no período pós-parto e seu reflexo na produção de leite de vacas de corte mantidas em pastagem nativa, Cerdótes et al. (2004) relataram maior produção para as vacas suplementadas que para as nãosuplementadas $(3,85$ vs. 3,25 L/dia) até os 63 dias, representando diferença de $18 \%$. Trabalhando com diferentes lotações animais ( 0,6 e 0,8 vaca/ha), Quadros \& Lobato (1997) verificaram que vacas Hereford e mestiças Hereford apresentaram maior produção quando a lotação foi menor (6,39 vs. 5,52 kg/dia).

Houve decréscimo mais acentuado na produção de leite entre o início (14 dias) e final da lactação (90 dias), para PN (4,52 vs. 3,25 L/dia), enquanto os valores de PNC indicaram flutuação, em decorrência do aumento na produção dos 70 para os 90 dias de lactação. As diferenças percentuais na redução da produção foram de 39 e 27\%, respectivamente (Tabela 1).

Diversos estudos demonstraram que o comportamento da produção de leite ao longo do período de lactação pode ocorrer de diferentes maneiras, podendo ser quadrático, como comumente verificado em bovinos leiteiros, ou linear decrescente (Lamond et al., 1969; Bond \& Wiltbank, 1970; Leal \& Freitas, 1982; Alencar et al., 1988; Restle et al., 2003). Analisando estes trabalhos, nota-se que a condição alimentar a que os animais são submetidos, sobretudo a quantidade e qualidade da pastagem,é o componente que mais influencia este comportamento. principalmente, a quantidade e qualidade da pastagem. Outros estudos apontam menor persistência na lactação quando em piores condições nutricionais. Provavelmente

Tabela 1 - Médias para produções diária e total de leite (L), de acordo com o sistema de alimentação e o dia de lactação Table 1 - Means for daily and total milk yield (L), according to feeding system and lactation day

\begin{tabular}{|c|c|c|c|c|c|c|}
\hline \multirow[t]{2}{*}{$\begin{array}{l}\text { Sistema de alimentação } \\
\text { Feeding system }\end{array}$} & \multicolumn{4}{|c|}{$\begin{array}{l}\text { Dia de lactação } \\
\text { Lactation day }\end{array}$} & \multirow[t]{2}{*}{$\begin{array}{l}\text { Média } \\
\text { Mean }\end{array}$} & \multirow[t]{2}{*}{$\begin{array}{l}\text { Total } \\
\text { Total }\end{array}$} \\
\hline & 14 & 42 & 70 & 90 & & \\
\hline $\begin{array}{l}\text { Pastagem nativa }(\mathrm{PN}) \\
\text { Native pasture }\end{array}$ & $4,52 \mathrm{~B}$ & $4,41 \mathrm{~A}$ & $3,72 \mathrm{~A}$ & $3,25 \mathrm{~B}$ & $3,93 \mathrm{~B}$ & $291,4 \mathrm{~B}$ \\
\hline $\begin{array}{l}\text { Pastagem nativa mais cultivada (PNC) } \\
\text { Native plus cultivated pasture }\end{array}$ & $6,52 \mathrm{~A}$ & $5,44 \mathrm{~A}$ & $4,52 \mathrm{~A}$ & $5,11 \mathrm{~A}$ & $5,46 \mathrm{~A}$ & $401,7 \mathrm{~A}$ \\
\hline $\begin{array}{l}\text { Probabilidade } \mathrm{F} \\
\text { F probability }\end{array}$ & 0,0115 & 0,1876 & 0,0880 & 0,0009 & 0,0032 & 0,0043 \\
\hline
\end{tabular}

A, B Médias seguidas por letras maiúsculas diferentes na coluna, para o mesmo dia de lactação, diferem $(P<0,05)$ pelo teste $F$. $A, B$ Means followed by different capital letters in the column, for the same lactation day, differ $(P<0.05)$ by $F$ test. 
em razão da prioridade do aproveitamento da energia disponível, que, em condições adversas de alimentação, será disponibilizada em último momento para produção de leite. Estudando diferentes seqüências de alimentação com pastagem nativa e/ou cultivada de aveia + azevém, Ribeiro (1989) verificou redução linear na produção de leite dos 14 aos 126 dias de lactação. Segundo o autor, este resultado foi reflexo da baixa disponibilidade de MS/ha, em virtude dos reduzidos índices de precipitação pluviométrica ocorridos. Avaliando a produção de leite dos 14 aos 182 dias, Restle et al. (2003) verificaram comportamento quadrático da produção de leite de vacas mantidas em pastagem cultivada de aveia + azevém e capimpapuã, enquanto, para as vacas mantidas em pastagem nativa, houve queda linear. Segundo os autores, este resultado foi reflexo da maior concentração de matéria orgânica digestível e de proteína bruta da pastagem cultivada. Cerdótes et al. (2004) avaliaram a produção de leite de vacas suplementadas ou não com farelo de arroz integral a $0,7 \%$ do peso vivo e constataram redução linear na produção de leite, que passou de 4,25 L, aos 21 dias, para 2,95 L, aos 63 dias. Porém, o declínio da produção foi menor para as vacas suplementadas (23\%) que para as nãosuplementadas $(38 \%)$.
Os valores médios e a heterose referentes à produção de leite, de acordo com o grupo genético da vaca, o sistema de acasalamento e o dia de lactação, são apresentados na Tabela 2.

Não houve diferença entre grupos genéticos da vaca dentro do sistema de acasalamento, para nenhum dos dias de lactação avaliados, bem como para produções média e total de leite. Entre vacas $\mathrm{CH}$ e $\mathrm{NE}$, poderia ser esperada diferença na produção de leite, uma vez que as primeiras são de maior porte, o que é comprovado por diversos estudos, como os de Alencar et al. (1993) e Cruz et al. (1997), ao avaliarem as raças Canchim e Nelore, e Ribeiro et al. (1991), trabalhando com vacas Charolês e Aberdeen Angus. Segundo Restle et al. (2003), provavelmente o aporte nutricional disponível nos tipos de pastagens pode não ter sido suficientemente adequado para atender às maiores exigências nutricionais das vacas Charolês (de maior porte), impedindo-as de expressarem todo seu potencial para produção de leite em relação às Nelore (de porte médio). Segundo Ferrel \& Jenkins (1985), MontanoBermudez et al. (1990) e NRC (1996), existe associação positiva entre desempenho e exigências energéticas para mantença e produção, de modo que genótipos maiores e/ou selecionados para

Tabela 2 - Médias e heterose para produções diária e total de leite (L), de acordo com o grupo genético da vaca, o sistema de acasalamento e o dia de lactação

Table 2 - Means and heterosis for daily and total milk yield (L), according to cow genetic group, breeding system and lactation day

\begin{tabular}{|c|c|c|c|c|c|c|}
\hline \multirow[t]{2}{*}{$\begin{array}{l}\text { Sistema de acasalamento } \\
\text { Breeding system }\end{array}$} & \multicolumn{4}{|c|}{$\begin{array}{c}\text { Dia de lactação } \\
\text { Lactation day }\end{array}$} & \multirow[t]{2}{*}{$\begin{array}{l}\text { Média } \\
\text { Mean }\end{array}$} & \multirow[t]{2}{*}{$\begin{array}{l}\text { Total } \\
\text { Total }\end{array}$} \\
\hline & 14 & 42 & 70 & 90 & & \\
\hline \multicolumn{7}{|l|}{ Puras (Straightbreds) } \\
\hline $\begin{array}{l}\text { Charolês (Charolais) (CH) } \\
\text { Nelore (Nellore) (NE) } \\
\text { Média puras } \\
\text { Straightbreds mean }\end{array}$ & $\begin{array}{c}5,10 \\
4,42 \\
4,76 \mathrm{~B}\end{array}$ & $\begin{array}{c}4,20 \\
3,68 \\
3,94 \mathrm{~B}\end{array}$ & $\begin{array}{c}2,98 \\
3,23 \\
3,11 \mathrm{~B}\end{array}$ & $\begin{array}{c}3,31 \\
3,81 \\
3,56 \mathrm{~B}\end{array}$ & $\begin{array}{c}3,98 \\
3,72 \\
3,85 \mathrm{~B}\end{array}$ & $\begin{array}{c}285,7 \\
275,1 \\
280,4 \mathrm{~B}\end{array}$ \\
\hline \multicolumn{7}{|l|}{ Mestiças F1 (F1 Crossbreds) } \\
\hline $\begin{array}{l}1 / 2 \mathrm{CH}^{1} / 2 \mathrm{NE} \\
1 / 2 \mathrm{NE} 1 / 2 \mathrm{CH} \\
\text { Média } \mathrm{F} 1 \\
\text { F1 mean }\end{array}$ & $\begin{array}{c}6,55 \\
5,99 \\
6,27 \mathrm{~A}\end{array}$ & $\begin{array}{c}6,86 \\
4,96 \\
5,91 \mathrm{~A}\end{array}$ & $\begin{array}{c}5,37 \\
4,89 \\
5,13 \mathrm{~A}\end{array}$ & $\begin{array}{c}4,54 \\
5,07 \\
4,80 \mathrm{~A}\end{array}$ & $\begin{array}{c}5,89 \\
5,20 \\
5,55 \mathrm{~A}\end{array}$ & $\begin{array}{c}435,7 \\
389,9 \\
412,8 \mathrm{~A}\end{array}$ \\
\hline $\begin{array}{l}\text { Heterose, } \% \\
\text { Heterosis, } \%\end{array}$ & 31,7 & 50,0 & 65,0 & 34,8 & 44,2 & 47,2 \\
\hline $\begin{array}{l}\text { Probabilidade } \mathrm{F} \\
\text { F probability }\end{array}$ & 0,0566 & 0,0106 & 0,0002 & 0,0205 & 0,0017 & 0,0027 \\
\hline
\end{tabular}


rápido ganho de peso e com maior potencial para produção de leite apresentam maiores exigências.

Analisando o sistema de acasalamento, vacas cruzadas F1 produziram maior quantidade de leite durante toda a lactação, com valores de heterose expressivos oscilando de 31,7\% (14 dias) a 65,0\% (70 dias). Na média, a produção diária de leite foi de $44,2 \%$ (5,55 vs. $3,85 \mathrm{~L} /$ dia) e total, de 47,2\% (412,8 vs. 280,4 L), o que pode estar relacionado à herdabilidade da característica, pois, conforme demonstrado nos estudos de Koger (1973), Restle et al. (1995) e Restle et al. (2001), que avaliaram o desempenho em confinamento de animais puros e cruzados, há relação inversa entre herdabilidade e heterose.

Poucos são os trabalhos que avaliaram a heterose para produção de leite. Em estudo sobre a produção de leite de vacas de corte até os 150 dias de lactação, Daley et al. (1987) verificaram heterose significativa aos $105(0,98 \mathrm{~kg} / \mathrm{dia})$ e $150 \mathrm{dias}(1,38 \mathrm{~kg} / \mathrm{dia}) \mathrm{de}$ lactação, ao compararem o cruzamento de Hereford Red Poll. Os resultados de Cundiff et al. (1974) comprovam que vacas mestiças Bos taurus produziram mais leite que as vacas puras, resultando em heterose de $1 \%$ aos 14 dias e 7,5\% aos 42 dias de lactação. Notter et al. (1978) verificaram heterose significativa aos 128 dias e declínio com o avanço dos dias de lactação. A heterose declinou de 33\% (128 dias) para $10 \%$ (184 dias) e, na média, foi de $15 \%$.

Contudo, diversos trabalhos indicam maior produção de leite em vacas mestiças, além de diferenças entre os genótipos de animais mestiços, reflexo do grau de heterozigose e da ação aditiva dos gens. Reynolds et al. (1978) relataram que vacas Brangus produziram mais leite que vacas Angus, Brahman ou Africânder x Angus. Cundiff et al. (1974) reportaram que vacas Brahman x Angus e Brahman x Hereford de três anos de idade apresentaram maior produção de leite corrigida para 12 horas que vacas cruzas Hereford x Angus. Todd et al. (1968) verificaram que vacas Brahman $x$ Hereford produziram mais leite que as puras Brahman ou Hereford. Avaliando o potencial de produção de leite de vacas de quatro anos de idade de diferentes composições raciais de Hereford, Angus e Simental, acasaladas com touros Tarentaise ou Charolês, Kress et al. (1990) observaram que a produção de leite foi similar aos 40 dias, mas superior para as vacas $1 / 2$ Hereford $1 / 2$ Simental $(10,2 \mathrm{~kg} /$ dia $)$ e $1 / 4$ Hereford $3 / 4$ Simental $(11,1 \mathrm{~kg} /$ dia $)$ em relação às Hereford puras $(7,2 \mathrm{~kg} / \mathrm{dia})$. Em condições sul-brasilei- ras, Cerdótes et al. (2004) reportaram que, até os 63 dias de lactação, vacas mestiças fillhas de touros $\mathrm{CH}$ apresentaram maior produção de leite $(4,17 \mathrm{~L} /$ dia) que as puras $\mathrm{CH}$ e NE, porém, não diferiram significativamente das mestiças filhas de touros NE $(3,76 \mathrm{~L} /$ dia $)$.

Os valores médios referentes à persistência da lactação, de acordo com o grupo genético da vaca e sistema de acasalamento, encontram-se na Tabela 3.

Houve efeito do grupo genético da vaca para persistência da lactação, e, na comparação entre as puras, vacas NE foram as que apresentaram menor

Tabela 3 - Médias e heterose para persistência da lactação (mL/dia), de acordo com o grupo genético da vaca e o sistema de acasalamento

Table 3 - Means and heterosis for lactation persistency ( $\mathrm{mL}$ / day), according to cow genetic group and breeding system

Sistema de acasalamento Persistência da lactação*, $\mathrm{mL} /$ dia Breeding system Lactation persistency, $m L / d a y$

Puras (Straightbreds)

Charolês (Charolais) (CH) - $\quad-29$ a

Nelore (Nellore) (NE)

$-8 b$

Média puras

$-19 B$

Straightbreds mean

Mestiças F1 (F1 Crossbreds)

$1 / 2 \mathrm{CH}^{1} 1 / 2 \mathrm{NE}-47 \mathrm{a}$

$1 / 2 \mathrm{NE}^{1 / 2} \mathrm{CH}$

Média F1 -35A

F1 mean

Heterose, $\%$

Heterosis, \%

Probabilidade F para sistema $\quad 0,1001$

de acasalamento

F probability for breeding system

Probabilidade F para grupo

genético da vaca

F probability for cow genetic group

* Persistência da lactação = (produção de leite do último dia de lactação $[A]$ - maior produção de leite $[B]$ )/dias (A-B) (adaptado de Jenkins et al., 2000).

a,b Médias, na coluna, seguidas de letras minúsculas diferentes, na comparação entre $\mathrm{CH}$ e $\mathrm{NE}$ dentro do sistema de acasalamento de puras e entre $1 / 2 \mathrm{CH} 1 / 2 \mathrm{NE}$ e $1 / 2 \mathrm{NE} 1 / 2 \mathrm{CH}$ dentro do sistema de acasalamento de $F 1$, são diferentes $(P<0,1001)$ pelo teste Tukey.

A, BMédias, na coluna, seguidas por letras maiúsculas diferentes, na comparação entre puras e $F 1$, são diferentes $(P<0,05)$ pelo teste $\mathrm{F}$.

* Lactation persistency $=($ milk yield last day lactation $[A]-$ higher milk yield [B]) / days [A-B]) (adaptated from Jenkins et al., 2000).

$a, b$ Means, in a column followed by different small letters, for the comparison between $\mathrm{CH}$ and $\mathrm{NE}$ within the straightbreds breeding system and between $1 / 2 \mathrm{CH}^{1} 1 / 2 \mathrm{NE}$ and $1 / 2 \mathrm{NE} 1 / 2 \mathrm{CH}$ within the $\mathrm{F} 1$ system are different $(P<.1001)$ by Tukey test.

$A, B$ Means, in a column, followed by different capital letters, for the comparison between straightbreds and $F 1$, are different $(P<0.05)$ by $F$ test. 
redução na produção de leite ao longo do período de lactação (-8 vs. $-29 \mathrm{~mL} /$ dia), diferença de $262,5 \%$. Entre as mestiças F1, a menor redução foi verificada para as vacas filhas de touros NE (-23 vs. $-47 \mathrm{~mL} /$ dia), representando diferença de 104,3\%. Verifica-se, ainda, diferença significativa entre sistema de acasalamento, de modo que as vacas mestiças F1 foram as que apresentaram maior redução na produção de leite até os 90 dias de lactação (-35 vs. $-19 \mathrm{~mL} /$ dia), conferindo expressiva heterose de $84,2 \%$.

O grupo genético do touro teve influência na persistência da lactação, atuando tanto nas vacas puras como nas mestiças F1. Vacas filhas de touros NE apresentaram declínio menos acentuado na produção de leite. Em estudo realizado por Jenkins et al. (2000), que avaliaram a produção de leite de vacas F1 resultantes do acasalamento de vacas Angus e Hereford com touros Hereford, Angus, Shorthorn, Galloway, Longhorn, Nelore e Salers, foi verificado efeito significativo da raça do pai da vaca para persistência da lactação, sendo $-42 \mathrm{~g} /$ dia a persistência para vacas $\mathrm{F} 1$ filhas de touros NE. Avaliando o efeito da composição racial paterna (Hereford, Angus, Belgian Blue, Piemontês, Brahman, Boran ou Tuli) sobre a produção de leite da progênie, Freetly \& Cundiff (1998) relataram diferenças na produção de leite de acordo com o grupo genético do touro. Dos 50 aos 200 dias, o declínio na produção de leite foi menor nas novilhas filhas de touros Boran (10\%) e Brahman (19\%), e, nos demais grupos genéticos, oscilou entre 28 e $48 \%$. No estudo de Cerdótes et al. (2004), que avaliaram a produção de leite de vacas $\mathrm{CH}$, NE, mestiças filhas de touros $\mathrm{CH}$ e mestiças filhas de touros NE dos 21 aos 63 dias de lactação, as reduções na produção verificadas foram de 84, 37, 25 e $23 \%$, respectivamente, confirmando novamente o efeito marcante do genótipo NE na persistência da lactação. No trabalho de Restle et al. (2003), que estudaram comportamento da produção de leite ao longo de 182 dias de lactação, constatou-se que vacas $\mathrm{CH}$ tiveram sua produção de leite reduzida em 19\% até os 98 dias, enquanto, nas NE, houve aumento de $9 \%$.

O comportamento da produção de leite ao longo dos dias de lactação, de acordo com o grupo genético da vaca, pode ser melhor visualizado na Figura 1.

O declínio menos acentuado na produção de leite das vacas filhas de touros NE (puras e cruzadas) foi causado pelo pequeno aumento na produção a partir dos 70 dias de lactação. Minick et al.(2001), trabalhando

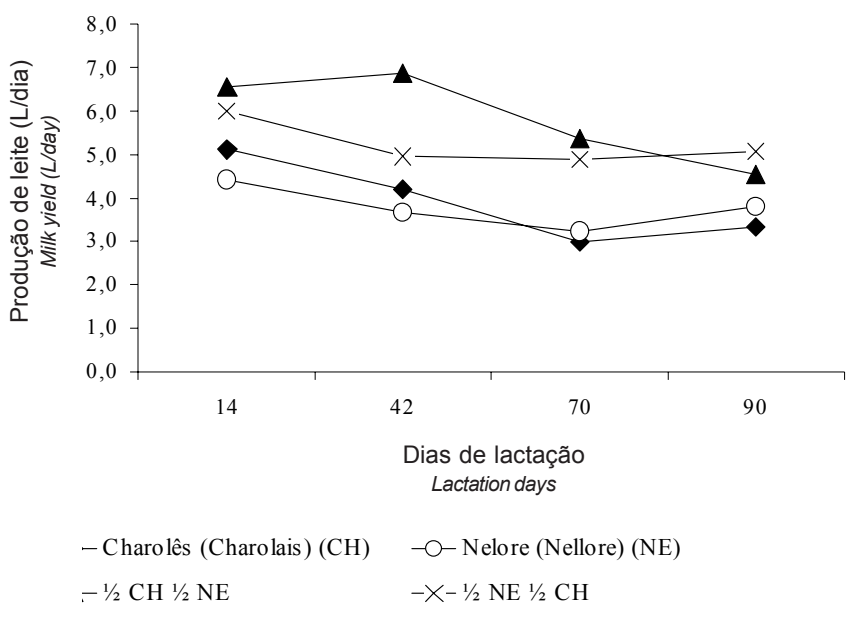

Figura 1 - Produção de leite (L/dia), de acordo com o grupo genético da vaca e dias de lactação.

Figure 1 - Milk yield (L/day), according to cow genetic group and lactation days.

com animais mestiços, e Johnson et al. (2003), com animais Brangus, verificaram que vacas mais produtivas tenderam a apresentar maiores declínios na produção após atingirem o pico de produção de leite. Estes resultados corroboram os deste estudo, em que a produção de leite das vacas $1 / 2 \mathrm{CH} \frac{1}{2} \mathrm{NE}$, que foram mais produtivas até os 42 dias e após, apresentou declínio acentuado. Os demais grupos genéticos demonstraram incremento na produção dos 70 aos 90 dias.

$\mathrm{Na}$ Tabela 4, encontram-se as médias e a heterose para produção de leite, de acordo com os sistemas de alimentação e acasalamento e os dias de lactação.

Houve diferença significativa para produção média diária de leite entre sistemas de acasalamento em todos os dias de lactação avaliados, nas vacas mantidas exclusivamente em pastagem nativa, verificando-se elevados valores de heterose, que oscilaram entre $65,3 \%$ (90 dias) e 139,7\% (70 dias). Na média, a diferença de produção entre vacas mestiças F1 e puras foi de $92,4 \%$ (5,18 vs. 2,69L/dia), e, para produção total de leite, foi de $98,2 \%$ (387,3 vs. 195,4 L).

Para o sistema de alimentação que incluiu pastagem cultivada (PNC), verifica-se que as médias obtidas entre animais puros e mestiços apresentaram razoável diferença numérica. Apesar de os valores de heterose terem sido positivos, não foram estatisticamente significativos 
Tabela 4 - Médias e heterose para produção diária e total de leite (L), de acordo com os sistemas de alimentação e de acasalamento e o dia de lactação

Table 4 - Means and heterosis for daily and total milk yield (L), according to feeding system, breeding system and lactation day

\begin{tabular}{|c|c|c|c|c|c|c|}
\hline \multirow[t]{2}{*}{$\begin{array}{l}\text { Sistema de alimentação } \\
\text { Feeding system }\end{array}$} & \multicolumn{4}{|c|}{$\begin{array}{l}\text { Dia de lactação } \\
\text { Lactation day }\end{array}$} & \multirow[t]{2}{*}{$\begin{array}{l}\text { Média } \\
\text { Mean }\end{array}$} & \multirow[t]{2}{*}{$\begin{array}{l}\text { Total } \\
\text { Total }\end{array}$} \\
\hline & 14 & 42 & 70 & 90 & & \\
\hline \multicolumn{7}{|l|}{ Past. nativa (PN) (Native pasture) } \\
\hline Puras (Straightbreds) & $3,38 \mathrm{~B}$ & $2,95 \mathrm{~B}$ & $2,19 \mathrm{~B}$ & $2,45 \mathrm{~B}$ & $2,69 \mathrm{~B}$ & $195,4 \mathrm{~B}$ \\
\hline Mestiças F1 (F1 Crossbreds) & $5,66 \mathrm{~A}$ & $5,86 \mathrm{~A}$ & $5,25 \mathrm{~A}$ & $4,05 \mathrm{~A}$ & $5,18 \mathrm{~A}$ & $387,3 \mathrm{~A}$ \\
\hline Heterose PN, \% (heterosis, \%) & 67,5 & 98,6 & 139,7 & 65,3 & 92,4 & 98,2 \\
\hline $\begin{array}{l}\text { Probabilidade } \mathrm{F}^{*} \\
\text { F probability } *\end{array}$ & 0,0997 & 0,0239 & 0,0006 & 0,1208 & 0,0107 & 0,0058 \\
\hline \multicolumn{7}{|l|}{$\begin{array}{l}\text { Past. nativa }+ \text { cultivada }(\mathrm{PNC}) \\
\text { Native }+ \text { cultivated pasture }\end{array}$} \\
\hline Puras (Straightbreds) & $6,15 \mathrm{~A}$ & $4,93 \mathrm{~A}$ & $4,02 \mathrm{~B}$ & $4,67 \mathrm{~A}$ & $5,01 \mathrm{~A}$ & $365,3 \mathrm{~B}$ \\
\hline Mestiças F1 (F1 Crossbreds) & $6,89 \mathrm{~A}$ & $5,95 \mathrm{~A}$ & $5,01 \mathrm{~A}$ & $5,56 \mathrm{~A}$ & $5,92 \mathrm{~A}$ & $438,2 \mathrm{~A}$ \\
\hline $\begin{array}{l}\text { Probabilidade } \mathrm{F} * * \\
\text { F probability } * *\end{array}$ & 0,3974 & 0,2095 & 0,0739 & 0,1753 & 0,1390 & 0,0958 \\
\hline
\end{tabular}

* Para sistema de acasalamento no sistema de alimentação PN (for breeding system on the feeding system PN).

** Para sistema de acasalamento no sistema de alimentação PNC (for breeding system on the feeding system PNC).

A, B Médias, na coluna, seguidas por letras maiúsculas diferentes, na comparação entre puras e $F 1$, são diferentes $(P<0,1208)$ pelo teste F.

A, B Means, in a column, followed by different capital letters, for the comparison between straightbreds and $F 1$, are different $(P<0.1208)$ by $F$ test.

entre os dias de lactação, com exceção dos 70 dias $(24,6 \%)$. Contudo, analisando a produção total de leite, verificou-se que vacas cruzadas $\mathrm{F} 1$ foram $20 \%$ mais produtivas que as puras (438,2 vs. 365,3 L).

Os valores de heterose (Tabela 4) comprovam que houve maior distanciamento entre genótipos puros e cruzados quando as condições nutricionais foram baixas. Esta diferença diminuiu quando o nível nutricional melhorou, demonstrando que os genótipos puros são mais sensíveis à variação do meio ambiente. Além disso, é provável que os animais mestiços tenham consumido mais alimento para preencherem suas maiores exigências nutricionais. Estudos realizados por Restle \& Alves Filho (1992) com animais na fase de recria em confinamento, Koger (1973), Casaccia et al. (1993), Restle et al. (1995) e Restle et al. (1999b), na fase de terminação em confinamento, demonstraram superioridade dos animais cruzados em relação aos puros para consumo de matéria seca (kg). No estudo de Deutscher \& Whiteman (1971), vacas mestiças produziram mais leite em todos os períodos avaliados, indicando que estas são capazes de produzir mais leite em condições de baix a condição nutricional que as puras. Contudo, os índices reprodutivos foram prejudicados. Avaliando a relação entre o grupo genético da vaca (Angus, Brahman e cruzas recíprocas) em diferentes sistemas forrageiros, Brown \& Brown Jr. (2002) verificaram heterose similar para produção de leite entre os sistemas avaliados (Bermuda, Festuca ou Rotação Bermuda x Festuca), que, em média, foi de $2,2 \mathrm{~kg} / \mathrm{dia}$. Em estudo mais recente, Johnson et al. (2003) concluíram que, para cada kg de leite a mais produzido, houve aumento de 0,33 e $0,37 \mathrm{~kg}$ no consumo de matéria seca de forragem durante o início e final da lactação, respectivamente.

$\mathrm{Na}$ Figura 2, podem ser visualizados os resultados da Tabela 4 ao longo do período de lactação. Houve expressivo incremento na produção de leite das vacas puras quando as condições alimentares foram melhoradas. Contudo, analisando as produções das vacas $\mathrm{F} 1$, as produções de leite foram muito semelhantes para ambos os sistemas de alimentação, o que demonstra que o cruzamento resultou em animais mais adaptados às condições ambientais adversas a que foram submetidos (pastagem nativa), sendo, portanto, mais eficientes e produtivos. Holloway et al. (1985) comentam em seu estudo, que o impacto do aumento da disponibilidade de nutrientes no incremento da produção de leite é resultado das diferenças entre os grupos genéticos nos requerimentos de energia para as várias 


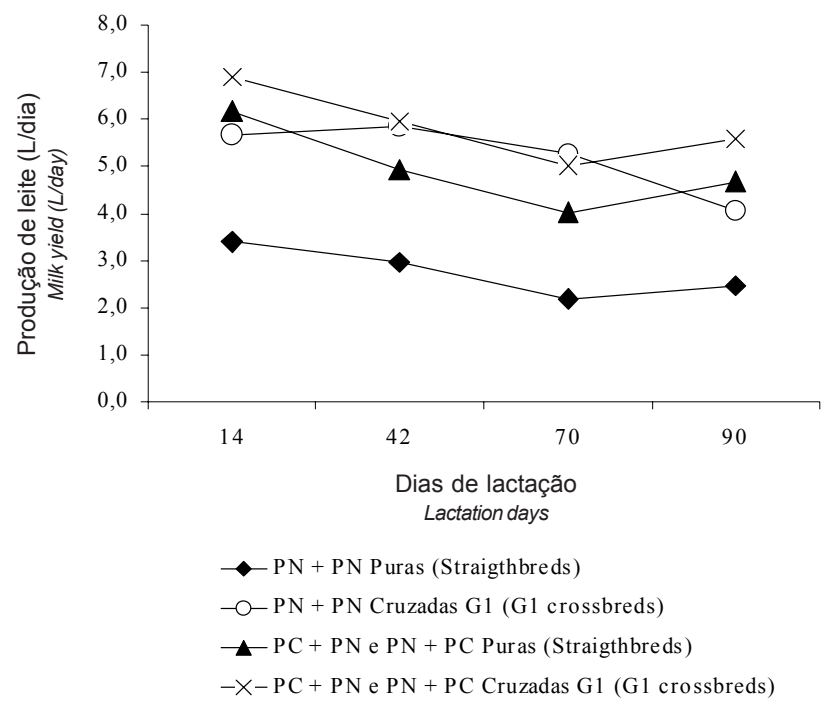

Figura 2 - Produção de leite (L/dia) de acordo com o sistema de alimentação, o sistema de acasalamento e os dias de lactação.

Figure 2 - Milk yield (L/day), according to feeding system, breeding system and lactation days.

funções corporais ou das diferenças na prioridade do uso de nutrientes. Os autores verificaram que vacas Angus e Hereford puras produziram mais leite com o aumento da disponibilidade de nutrientes que as vacas cruzas recíprocas F1, que depositaram mais gordura corporal.

\section{Conclusões}

Vacas mantidas em pastagem cultivada por períodos curtos produziram mais leite que as manejadas apenas em pastagem nativa e apresentam menor declínio na produção de leite com o avanço da lactação.

Em condições nutricionais baixas, houve maior distanciamento entre genótipos puros e mestiços, resultando em heterose elevada e diminuindo a diferença e a heterose quando o nível nutricional foi melhorado.

A persistência da lactação foi maior nas vacas filhas de touros Nelore que nas filhas de touros Charolês, assim como nas puras em relação às mestiças $F 1$.

Conforme os expressivos valores de heterose, vacas mestiças $\mathrm{F} 1$ foram mais produtivas que as puras, independentemente do sistema de alimentação utilizado, indicando efeito benéfico do cruzamento no incremento da produção de leite de vacas de corte.

\section{Literatura Citada}

ALBUQUERQUE, L.G.; ELER, J.P.; COSTA, M.J.R.P. Produção de leite e desempenho do bezerro na fase de aleitamento em três raças bovinas de corte. Revista Brasileira de Zootecnia, v.22, n.5, p.745-754, 1993.

ALENCAR, M.M.; OLIVEIRA, F.T.T.; TAMBASCO, A.J. et al. Desenvolvimento pós-desmama e eficiência reprodutiva pós-parto em gado de corte: Influência da produção de leite. Revista da Sociedade Brasileira de Zootecnia, v.22, n.6, p.1012-1018, 1993.

ALENCAR, M.M.; RUZZA, F.J.; PORTO, E.J.S. Desempenho produtivo de fêmeas das raças Canchim e Nelore. III. Produção de leite. Revista da Sociedade Brasileira de Zootecnia, v.17, n.4, p.317-328, 1988

ASSOCIATION OF OFFICIAL ANALYTICAL CHEMISTS AOAC. Official methods of analysis. 14.ed. Washington, D.C.: 1984. 1141p.

BOGGS, D.L.; SMITH, E.F.; SCHALLES, R.R. et al. Effects of milk and forage intake on calf performance. Journal of Animal Science, v.51, n.3, p.550-553, 1980.

BOND, J.; WILTBANK, J.N. Effect of energy and protein on estrus, conception rate, growth and milk production of beef females. Journal of Animal Science, v.30, n.3, p.438-444, 1970.

BROWN, M.A.; BROWN JR., A.H. Relationship of milk yield and quality to preweaning gain of calves from Angus, Brahman and reciprocal-cross cows on different forage systems. Journal of Animal Science, v.80, p.2522-2527, 2002.

CASACCIA, J.L.; PIRES, C.C.; RESTLE, J. Confinamento de bovinos inteiros ou castrados de diferentes grupos genéticos. In: REUNIÃO ANUAL DA SOCIEDADE BRASILEIRA DE ZOOTECNIA, 30, Rio de Janeiro, RJ. Anais... Rio de Janeiro: Sociedade Brasileira de Zootecnia, 1993. p.468.

CERDÓTES, L.; RESTLE, J.; ALVES FILHO, D.C. et al. Produção e composição do leite de vacas de quatro grupos genéticos submetidas a dois manejos alimentares no período de lactação. Revista Brasileira de Zootecnia, v.33, n.3, p.610-622, 2004.

CRUZ, G.M.; ALENCAR, M.M.; TULliO, R.R. Produção e composição do leite de vacas das raças Canchim e Nelore. Revista Brasileira de Zootecnia, v.26, n.5, p.887-893, 1997.

CUNDIFF, L.V.; GREGORY, K.E.; SCHWULST, F.J. Effects of heterosis on maternal performance and milk production in Hereford, Angus and Shorthorn cattle. Journal of Animal Science, v.38, n.4, p.728-745, 1974.

DALEY, D.R.; McCUSKEY, A.; BAILEY, C.M. Composition and yield of milk from beef-type Bos taurus and Bos indicus $\mathrm{x}$ Bos taurus dams. Journal of Animal Science, v.64, p.373-384, 1987.

DEUTSCHER, G.H.; WHITEMAN, J.V. Productivity as twoyear-olds of Angus-Holstein crossbreds compared to Angus heifers under range conditions. Journal of Animal Science, v.33, n.2, p.337-342, 1971 .

DUNN, T.G.; WILTBANK, J.N.; ZIMMERMAN, D.R. Dam's energy intake on milk production and calf gains. Journal of Animal Science, v.24, p.586 (Abstracts), 1965.

FERREL, C.L.; JENKINS, T.G. Cow type and the nutritional environment: nutritional aspects. Journal of Animal Science, v.61, p.725-741, 1985.

FREETLY, H.C.; CUNDIFF, L.V. Reproductive performance, calf growth, and milk production of first-calf heifers sired by 
seven breeds and raised on different levels of nutrition. Journal of Animal Science, v.76, p.1513-1522, 1998

HOLLOWAY, J.W.; BUTTS, W.T.; McCURLEY, J.R. et al. Breed $\mathrm{x}$ nutritional environment interactions for beef female weight and fatness, milk production and calf growth. Journal of Animal Science, v.61, n.6, p.1354-1363, 1985.

JENKINS, T.G.; FERREL, C.L.; ROBERTS, A.J. Lactation and calf weight traits of mature crossbred cows fed varying daily levels of metabolizable energy. Journal of Animal Science, v.78, p.7-14, 2000.

JOHNSON, C.R.; LALMAN, D.L.; BROWN, M.A. et al. Influence of milk production potential on forage dry matter intake by multiparous and primiparous Brangus females. Journal of Animal Science, v.81, p.1837-1846, 2003.

KOGER, M. Summary. In: KOGER, M.; CUNHA, T.J.; WARWICK, A.C. (Eds.) Crossbreeding beef cattle. Series 2. Gainesville: University of Florida Press, 1973. p.434-453.

KRESS, D.D.; DOOMBOS, D.E.; ANDERSON, D.C. Performance of crosses among Hereford, Angus and Simental cattle with different levels of Simmental Breeding: V. Calf production, milk production and reproduction of three- to eight-year-old dams. Journal of Animal Science, v.68, p.1910-1921, 1990.

LAMOND, D.R.; HOLMES, J.H.G.; HAYDOCK, K.P. Estimation of yield and composition of milk produced by grazing beef cows. Journal of Animal Science, v.29, p.606-611, 1969.

LEAL, T.C.; FREITAS, J.E. Correlação entre produção de leite e ganho de peso de bezerros da raça Charolesa. Anuário Técnico do IPZFO, v.9, p.91-101, 1982.

MELTON, A.A.; RIGGS, J.K.; NELSON, L.A. et al. Milk production, composition and calf gains of Angus, Charolais and Hereford cows. Journal of Animal Science, v.26, p.804-809, 1967.

MINICK, J.A.; BUCHANAN, D.S.; RUPERT, S.D. Milk production of crossbred daughters of high- and low-milk EPD Angus and Hereford bulls. Journal of Animal Science, v.79, p.1386-1393, 2001.

MONTANO-BERMUDEZ, M.; NIELSEN, M.K.; DEUTSCHER, G.H. Energy requirements for maintenance of crossbred beef cattle with different genetic potential for milk. Journal of Animal Science, v.68, p.2279-2288, 1990.

MORENO, J.A. Clima do Rio Grande do Sul. Porto Alegre: Secretaria da Agricultura, 1961. 42p.

NATIONAL RESEARCH COUNCIL - NRC. Nutrient requirements of beef cattle. 6.ed. Washington, D.C.: National Academy Press, 1996. 242p.

NOTTER, D.R.; CUNDIFF, L.V.; SMITH, G.M. et al. Characterization of biological types of cattle. VII. Milk production in young cows and transmitted and maternal effects on preweaning growth of progeny. Journal of Animal Science, v.46, n.4, p.908-921, 1978.

POLLI, V.A.; LOBATO, J.F.P. Utilização de pastagem temperada por diferentes categorias do rebanho. I. Vacas com cria. In: REUNIÃO ANUAL DA SOCIEDADE BRASILEIRA DE ZOOTECNIA, 22., Camboriú. Anais... Camboriú: Sociedade Brasileira de Zootecnia, 1985. p.503.

QUADROS, S.A.F.; LOBATO, J.F.P. et al. Efeito da lotação animal na produção de leite de vacas de corte primíparas e no desenvolvimento de seus bezerros. Revista Brasileira de Zootecnia, v.26, n.1, p.27-33, 1997.

RESTLE, J.; ALVES FILHO, D.C. Confinamento de terneiros inteiros ou castrados de diferentes grupos genéticos. In: REUNIÃO ANUAL DA SOCIEDADE BRASILEIRA DE ZOOTECNIA, 29., 1992, Lavras. Anais... Lavras: Sociedade Brasileira de Zootecnia, 1992, p.186.
RESTLE, J.; BRONDANI, I.L.; ALVES FILHO, D.C. et al. Efeito do grupo genético e heterose na terminação de vacas de descarte em confinamento. Revista Brasileira de Zootecnia, v.30, n.2, p.374-382, 2001.

RESTLE, J.; FELTEN, H.G.; VAZ, F.N. Efeito de raça e heterose para desempenho em confinamento de novilhos de corte. In: REUNIÓN LATINOAMERICANA DE PRODUCCIÓN ANIMAL, 14., 1995, Mar del Plata. Memórias... Balcarce: ALPA, 1995, v.3-4, p.852-854.

RESTLE, J.; PACHECO, P.S.; MOLETTA, J.L. et al. Grupo genético e nível nutricional pós-parto na produção e composição do leite de vacas de corte. Revista Brasileira de Zootecnia, v.32, n.3, p.585-597, 2003.

RESTLE, J.; PACHECO, P.S.; PASCOAL, L.L. et al. Efeito da pastagem, da produção e composição do leite no desempenho de bezerros de diferentes grupos genéticos. Revista Brasileira de Zootecnia, v.33, n.3, p.691-703, 2004.

RESTLE, J.; POLLI, V.A.; ALVES FILHO, D.C. et al. Desenvolvimento de bovinos de corte de diferentes grupos genéticos desmamados aos 3 ou 7 meses de idade. Revista Brasileira de Zootecnia, v.28, n.5, p.1023-1030, 1999b.

RESTLE, J.; POLLI, V.A.; SENNA, D.B. Efeito de grupo genético e heterose sobre a idade e peso à puberdade e sobre o desempenho reprodutivo de novilhas de corte. Pesquisa Agropecuária Brasileira, v.34, n.4, p.701-707, 1999a.

REYNOLDS, W.L.; DeROUEN, T.M.; BELLOWS, R.A. Relationships of milk yield of dam to early growth rate of straightbred and crossbred calves. Journal of Animal Science, v.47, n.3, p.584-594, 1978.

RIBEIRO, E.L.A. Influência de diferentes seqüências de pastagens na produção de leite e no desempenho de vacas de dois grupos genéticos. Santa Maria,: Universidade Federal de Santa Maria, 1989. 149p. Dissertação (Mestrado em Zootecnia) - Universidade Federal de Santa Maria, 1989.

RIBEIRO, E.L.A.; RESTLE, J. Desempenho de terneiros Charolês e Aberdeen Angus puros e seus mestiços com Nelore. Pesquisa Agropecuária Brasileira, v.26, n.8, p.1145-1151, 1991.

RIBEIRO, E.L.A.; RESTLE, J.; PIRES, C.C. Produção e composição do leite de vacas Charolês e Aberdeen Angus amamentando bezerros puros ou mestiços. Pesquisa Agropecuária Brasileira, v.26, n.8, p.1267-1273, 1991.

RIO GRANDE DO SUL. Secretaria da Agricultura. Departamento de Pesquisa. Instituto de Pesquisas Agronômicas. Observações Meteorológicas do Estado do Rio Grande do Sul. Boletim Técnico, n.3, 1979, 270p.

RUTLEDGE, J.J.; ROBISON, O.W.; AHLSCHWEDE, W.T. et al Milk yield and its influence on 205-day weight of beef calfs. Journal of Animal Science, v.33, p.563-567, 1971.

STATISTICAL ANALYSIS SYSTEMS - SAS. User's guide. Version 6, Cary: 1997. v.2, 1052p.

TILLEY, J.M.A.; TERRY, R.A. A two-stage technique for the in vitro digestion of forage crop. Journal of British Grassland Society, v.18, n.2, p.104-111, 1963.

TODD, J.C.; RIGGS, J.K.; SMITH, J.C. Milk yields and calf weights from Brahman, Hereford and crossbred cows in the Gulf Coast Prairie. Journal of Animal Science, v.27, p.286, 1968 (Abstract).

WILM, H.G.; COSTELlO, D.F.; KLIPPLE, G.E. Estimating forage yield by the double-sampling methods. Journal of American Society of Agronomy, v.36, p.194-203, 1944. 